University of South Carolina

Scholar Commons

$12-15-2009$

\title{
Enhance Diamond Coating Adhesion by Oriented Interlayer Microcracking
}

Habio Guo

Xingcheng Xiao

Yue Qi

Zhi-Hui Xu

University of South Carolina - Columbia

Xiaodong Li

University of South Carolina - Columbia, lixiao@cec.sc.edu

Follow this and additional works at: https://scholarcommons.sc.edu/emec_facpub

Part of the Applied Mechanics Commons, and the Other Mechanical Engineering Commons

\section{Publication Info}

Published in Journal of Applied Physics, Volume 106, Issue 12, 2009, pages \#123514-.

(C) Journal of Applied Physics 2009, American Institute of Physics.

Guo, H., Xiao, X., Qi, Y., Xu, Z-H., \& Li, X. (15 December 2009). Enhance Diamond Coating Adhesion by Oriented Interlayer Microcracking. Journal of Applied Physics, 106 (12), \#123514. http://dx.doi.org/ $10.1063 / 1.3267162$

This Article is brought to you by the Mechanical Engineering, Department of at Scholar Commons. It has been accepted for inclusion in Faculty Publications by an authorized administrator of Scholar Commons. For more information, please contact digres@mailbox.sc.edu. 


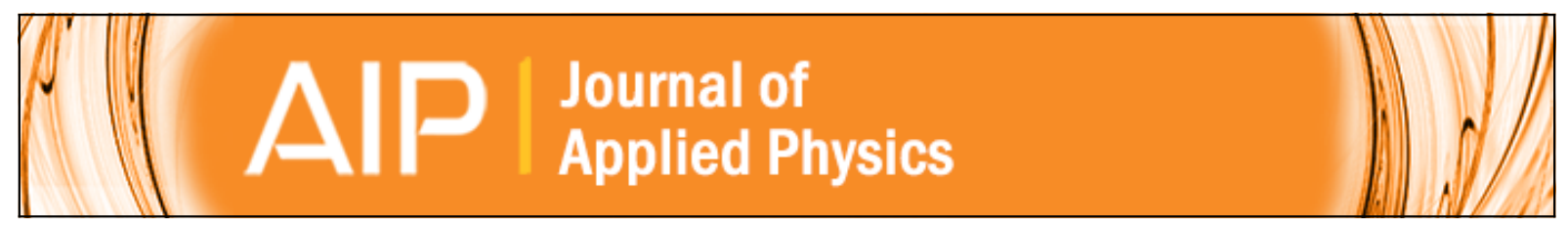

\section{Enhance diamond coating adhesion by oriented interlayer microcracking}

Haibo Guo, Xingcheng Xiao, Yue Qi, Zhi-Hui Xu, and Xiaodong Li

Citation: Journal of Applied Physics 106, 123514 (2009); doi: 10.1063/1.3267162

View online: http://dx.doi.org/10.1063/1.3267162

View Table of Contents: http://scitation.aip.org/content/aip/journal/jap/106/12?ver=pdfcov

Published by the AIP Publishing

Articles you may be interested in

The effect of interface adhesion on buckling and cracking of hard thin films

Appl. Phys. Lett. 105, 161912 (2014); 10.1063/1.4900443

Adhesion testing of atomic layer deposited $\mathrm{TiO} 2$ on glass substrate by the use of embedded $\mathrm{SiO} 2$ microspheres J. Vac. Sci. Technol. A 32, 01A102 (2014); 10.1116/1.4827197

Gold-titania interface toughening and thermal conductance enhancement using an organophosphonate nanolayer

Appl. Phys. Lett. 102, 201605 (2013); 10.1063/1.4807436

Atomistic mechanisms of moisture-induced fracture at copper-silica interfaces

Appl. Phys. Lett. 99, 133103 (2011); 10.1063/1.3622304

Comparison of the adhesion of diamond coatings using indentation tests and micro-Raman spectroscopy J. Appl. Phys. 86, 5509 (1999); 10.1063/1.371614

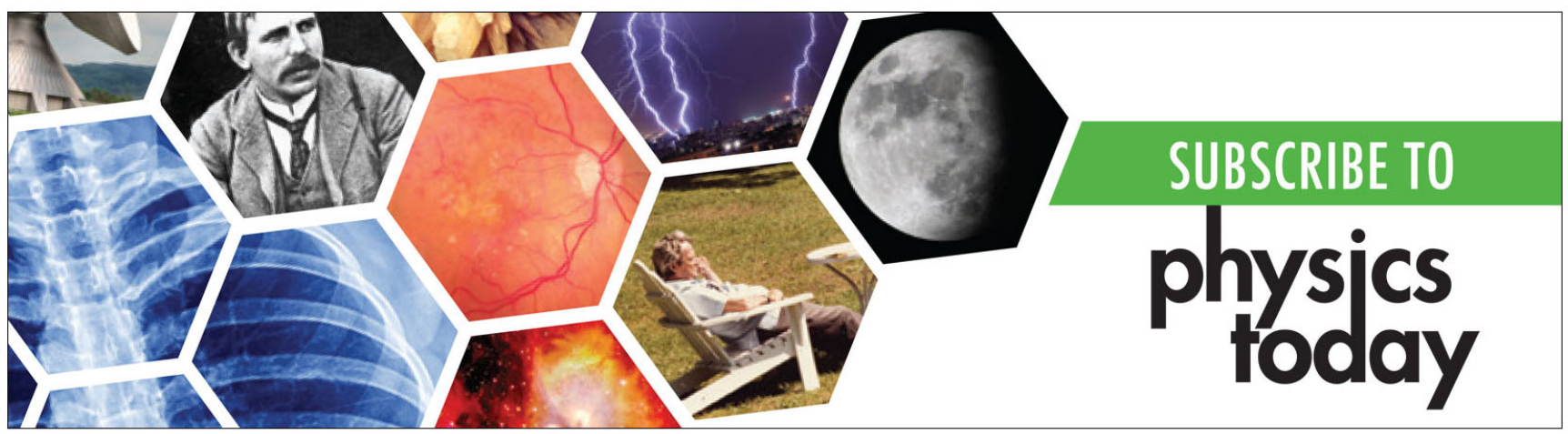




\title{
Enhance diamond coating adhesion by oriented interlayer microcracking
}

\author{
Haibo Guo, ${ }^{1}$ Xingcheng Xiao, ${ }^{2, a)}$ Yue Qi, ${ }^{2, b)}$ Zhi-Hui Xu, ${ }^{1}$ and Xiaodong $\mathrm{Li}^{1, \mathrm{c})}$ \\ ${ }^{1}$ Department of Mechanical Engineering, University of South Carolina, 300 Main Street, Columbia, \\ South Carolina 29208, USA \\ ${ }^{2}$ Materials and Processes Laboratory, General Motors R\&D Center, 30500 Mound Road, Warren, \\ Michigan 48090, USA
}

(Received 4 June 2009; accepted 31 October 2009; published online 18 December 2009)

\begin{abstract}
In this paper, we report a microcrack toughening mechanism for enhancing the adhesion of diamond coating. The oriented microcracks were formed within the TiC interlayer to dissipate strain energy and accommodate deformation via the crack opening-closing mechanism, thus enhancing the coating/substrate interfacial toughness. The delamination of diamond coating was effectively prevented when the parallel microcracks were confined within the interlayer and arrested at interfaces of coating/interlayer/substrate. Density functional theory calculations revealed that the highly anisotropic fracture strength of the TiC phase energetically favors crack initiation and propagation along (100) planes only, which are $54.7^{\circ}$ away from the interface. These microcracks are constrained inside the interlayer by the two strong interfaces in the substrate/interlayer/coating system. The new microcrack toughening mechanism with these combined features has a wide application to enhance the adhesion of thin-film coatings. (c) 2009 American Institute of Physics. [doi:10.1063/1.3267162]
\end{abstract}

\section{INTRODUCTION}

Most coatings (microelectromechanical systems, optical, decorative, and protective) are desired to adhere on the substrates during their applications to ensure their reliability and lifetime. However, delamination of thin-film coatings is responsible for the failure that occurs during those applications in many occasions. This problem is extraordinarily severe in chemical vapor deposited (CVD) diamond coatings, due to high residual stresses in coatings and weak coating/substrate interfaces. Significant progresses have been made in minimizing the coating residual stresses, including both reducing the deposition temperature to lower the thermal stress and engineering the structure of grain boundaries to release the intrinsic stress. ${ }^{1,2}$ However, it remains a great challenge to strengthen and toughen the interface of CVD diamond coating/substrate. In this paper, we present a new and versatile interface adhesion mechanism controlling the nucleation, growth, and alignment of multiple microcracks in the interlayer by utilizing the anisotropic fracture characteristics of interlayer materials. The opening-closing of these microcracks can additionally dissipate strain energy and accommodates deformation, thereby enhancing the overall adhesion.

The adhesion of a thin-film coating is typically characterized by the energy associated with the separation of the coating/substrate, i.e., the work of interfacial fracture, $\Gamma$. Enhancing the interfacial toughness comes through increasing the intrinsic work of adhesion and promoting the energy dissipation for the nonfracture processes. ${ }^{3,4}$ The intrinsic work of adhesion, $\Gamma_{0}$, is defined as the reversible energy needed to break the bonds at the interface: $\Gamma_{0}=\left(\gamma_{s}+\gamma_{f}\right)-\gamma_{s f}$, where $\gamma_{s}$ and $\gamma_{f}$ are the surface energies of the substrate and the coat-

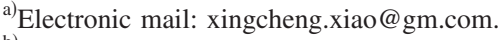

b) Electronic mail: yue.qi@gm.com.

${ }^{c)}$ Electronic mail: lixiao@engr.sc.edu.
}

ing, respectively, and $\gamma_{s f}$ is the interface energy. All the energy absorbed in the irreversible processes, such as plastic deformation, new interface creation, and interface roughening, can be included in $\Gamma_{i}$. Thus, the overall toughness is $\Gamma$ $=\Gamma_{0}+\Gamma_{i}{ }^{4}$ Based on these concepts, several approaches have been developed to improve the interfacial toughness and enhance the adhesion of diamond coating to certain substrates, such as avoiding the presence of deleterious elements at the substrate surface (increasing $\left.\Gamma_{0}\right)^{5}$ improving the seeding process (increasing $\Gamma_{0}$ ), or by forming an interlayer (increasing both $\Gamma_{0}$ and $\left.\Gamma_{i}\right){ }^{6}$

It has been well accepted that many carbide-forming metals favor diamond adhesion than noncarbide-forming metals due to the formation of carbide interlayers. ${ }^{7}$ Generally, carbide interlayers have been chosen to provide chemical bonds to both the diamond and the metal substrate, and also to alleviate abrupt changes of mechanical properties from coating to substrate. Ti is often used as an interlayer material, where titanium carbide (TiC) forms during the diamond deposition process because of the large negative heat of formation in the Ti-C system $\left[\Delta_{f}^{0} H_{\mathrm{TiC}}=-184.1 \mathrm{~kJ} \mathrm{~mol}^{-1}\right.$ (Ref. 8)]. The dominant directional Ti-C covalent bonds ${ }^{9-11}$ in TiC not only impart its high hardness to alleviate the difference between diamond and substate, ${ }^{12}$ but also impose brittleness and strong anisotropy in fracture toughness. ${ }^{13}$ The brittleness and anisotropy of the carbide interlayer could be utilized for energy dissipation, if multiple microcracks can be generated in a controlled manner, as we demonstrated in the following experiment and simulation.

\section{EXPERIMENTAL OBSERVATIONS OF PARALLEL CONFINED MICROCRACKS}

We directly deposited nanocrystalline diamond (NCD) coatings in a microwave plasma enhanced CVD system with the following conditions: $800 \mathrm{~W}$ microwave power, 150 Torr 


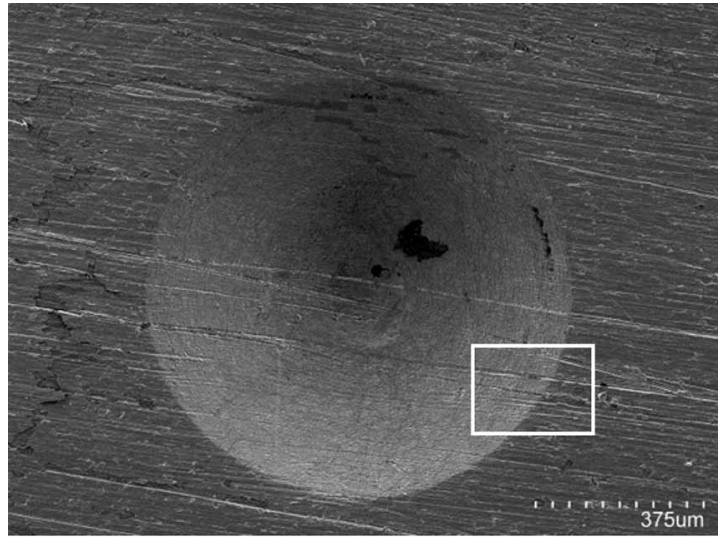

(a)

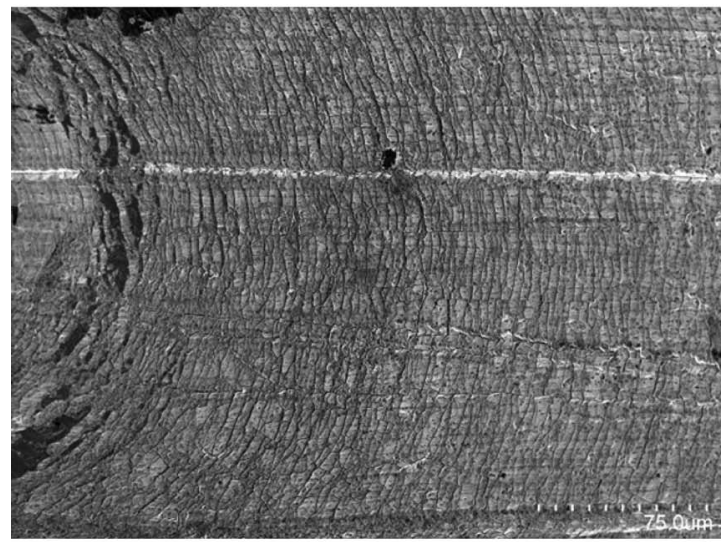

(b)

FIG. 1. SEM images of Rockwell C indent on diamond coated Ti substrate No delamination occurred around the indent, indicating the excellent adhesion of diamond to Ti substrate.

pressure, $600 \mathrm{sccm}$ total precursor flow rate, $1 \% \mathrm{CH}_{4}, 5 \%$ $\mathrm{H}_{2}$, and balance $\mathrm{Ar}$, and $600{ }^{\circ} \mathrm{C}$ deposition temperature. The total deposition time was $4 \mathrm{~h}$ and the resulting coating thickness is around 2 microns. All the diamond coatings were deposited on titanium foils with thickness around $0.25 \mathrm{~mm}$, which can easily generate large strain with low load in a micromechanical tester. We observed no delamination of the diamond coating under standard Rockwell $\mathrm{C}$ indentation tests with a load of $150 \mathrm{~kg}$, despite the large amount of cracks in the coating itself, as shown in Fig. 1.

In order to understand the exceptional strong adhesion of the diamond coating to titanium, we investigated the initiation and propagation of microcracks in the $\mathrm{TiC}$ interlayer with applying external load. A custom-built micromechanical tester integrated with an atomic force microscope (AFM) (Dimension 3100; Digital Instruments, Santa Barbara, CA, USA) was employed to perform three point bending tests on the coated samples. Meanwhile, the polished cross section surface was imaged simultaneously by the AFM under bending loading, as shown in Fig. 2(a). The micromechanical tester has a load resolution of $10 \mathrm{mN}$ and a displacement resolution of $1.6 \mu \mathrm{m}$. The AFM with silicon tip (5-10 nm radius) under tapping mode was used to scan the polished cross section surface to achieve higher lateral spatial resolution and less topographical distortions. A bending displace- ment was gradually applied to the sample with $0.012 \mathrm{~mm}$ increase each step and AFM images were correspondingly taken at each deformation stage.

Figure 2(b) showed an AFM image of the polished cross section surface before the bending test, where an interlayer lied between the diamond coating and the Ti substrate. In order to confirm the interlayer, we purposely peeled off NCD coating and exposed the interlayer for x-ray diffraction (XRD), which was performed on a Bruckers D8 Advanced XRD system with $\mathrm{Cu} \mathrm{K} \alpha$ radiation at $40 \mathrm{KV}$ and $20 \mathrm{~mA}$. We employed the glancing angle XRD technique with a glancing angle of $2^{\circ}$ to minimize the contribution from the Ti substrate. From the XRD pattern in Fig. 2(c), we identified the interlayer to be a $\mathrm{TiC}$ phase, which in fact formed during CVD growth of diamond coating at $600{ }^{\circ} \mathrm{C}$. The slight shift of diffraction peak to lower angle, comparing with the diffraction peaks from stress-free $\mathrm{TiC}$ powder, indicates the compressive stress presented in the $\mathrm{TiC}$ layer. Before the bending test, there was no crack in all the three layers (diamond coating, TiC interlayer, and Ti substrate), as shown in Fig. 2(b). When the bending displacement was increased to $0.144 \mathrm{~mm}$, cracks initiated and propagated only in the TiC interlayer, as shown in Fig. 2(d). It is interesting that all the cracks are aligned almost parallel to each other. The angles of the cracks (actually crack planes) relative to the interface were about $55^{\circ}$ with small variations due to the interface roughness. There was no linkage among those cracks. The propagation of cracks stopped when they reached both the interface between the diamond coating and $\mathrm{TiC}$ interlayer and the interface between $\mathrm{TiC}$ interlayer and the $\mathrm{Ti}$ substrate. No crack was observed in both the diamond coating and the Ti substrate under such bending displacement. Most importantly, the orientation of the generated microcracks was far away from the direction parallel to the interface, which in fact avoided the delamination of the coating from the substrate.

To further confirm that cracks occurred only in the TiC interlayer with the external loading, nanoindentation tests were performed on the cross section of the diamond/TiC/Ti [as shown in Fig. 3(a)]. A triboscope nanoindenter (Hysitron Inc. Minneapolis, MN, USA) with a Berkovich indenter was used to make indentations on the mechanically polished cross section of the diamond coating, $\mathrm{TiC}$ interlayer, and $\mathrm{Ti}$ substrate, respectively. Typical nanoindentation loaddisplacement curves were shown in Fig. 3(b). With the same indentation load, the penetration depth (displacement) was shallow on the hard diamond coating while deep on the soft Ti substrate. A clear pop-in (step) on the loading curve of the TiC interlayer was observed, which indicated that cracks occurred during indentation [as shown in Fig. 3(b)], leading to a sudden advance in indentation displacement. ${ }^{14,15}$ Further loading did not induce any additional initiation or propagation of indentation cracks in the $\mathrm{TiC}$ interlayer. The unloading curve of the $\mathrm{TiC}$ interlayer, which was different from those of the diamond coating and Ti, exhibited a "pushing back" at the lower part of the unloading curve. This pushing back resulted from the closing of the open indentation cracks 


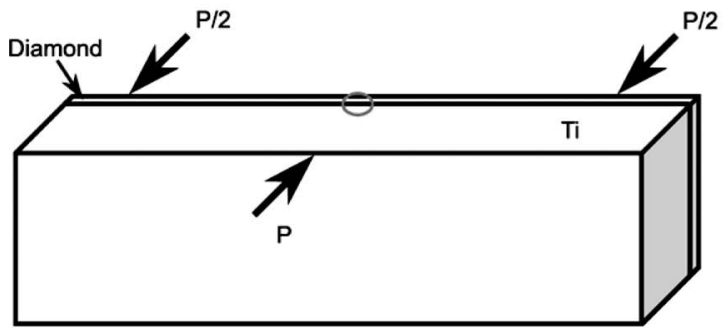

(a)

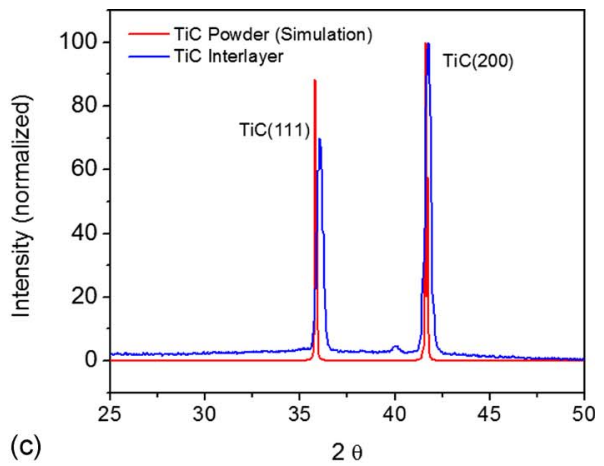

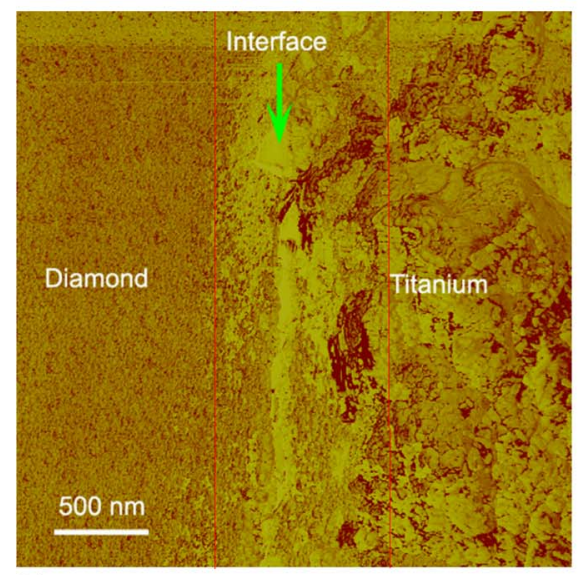

(b)

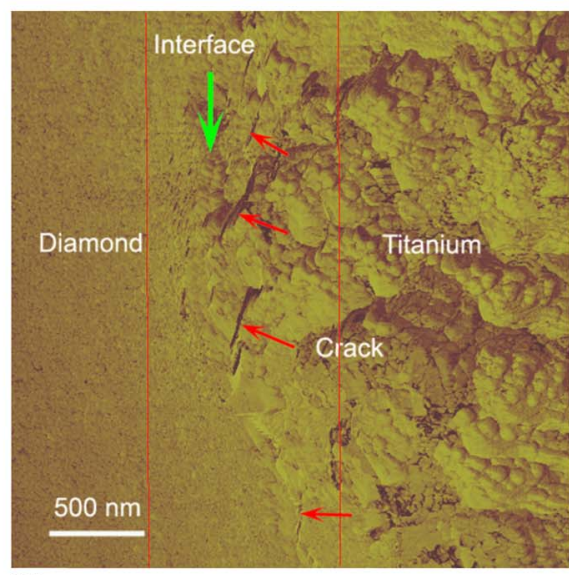

(d)

FIG. 2. (Color online) Three-point bending test of diamond coated Ti sample. (a) Schematic of three-point bending. AFM observation was performed at the TiC interlayer (circled area) between diamond coating and Ti substrate; (b) AFM image of the TiC interlayer before bending; (c) XRD of TiC interlayer formed during diamond deposition; (d) AFM image of the TiC interlayer with a $0.144 \mathrm{~mm}$ bending displacement. Visible cracks are marked by small arrows.

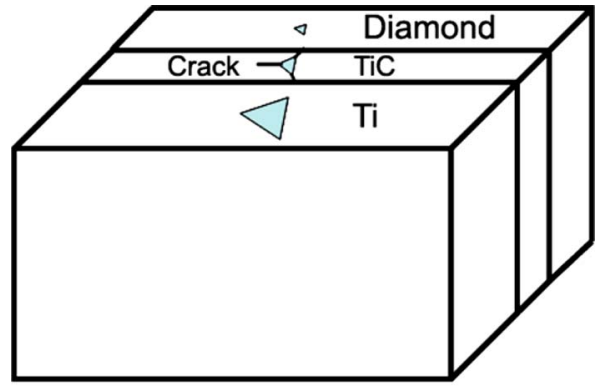

(a)

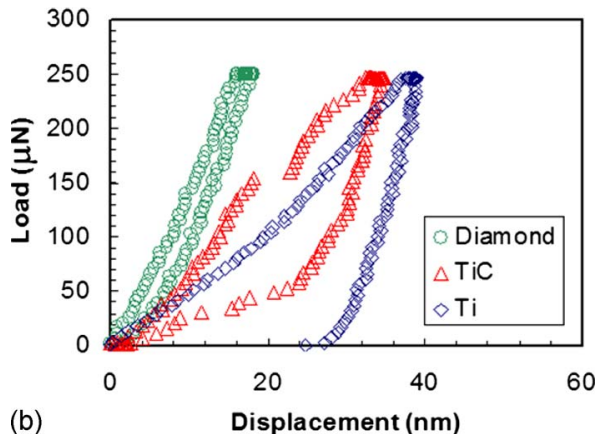

FIG. 3. (Color online) Nanoindentations on the cross section of the diamond coated Ti sample. (a) Schematic of nanoindentation test on diamond coating, $\mathrm{TiC}$ interlayer, and $\mathrm{Ti}$ substrate; (b) Typical nanoindentation loaddisplacement curves of diamond coating, TiC interlayer, and Ti substrate. when the load was released. Such crack opening-closing processes were expected to release strain energy by accommodating the deformation.

The agreement between the AFM three-point bending and nanoindentation experiments confirmed that the $\mathrm{TiC}$ interlayer was brittle. However, the orientation of the microcracks which were orientated away from the interface and isolated from each other, actually avoided the formation of larger cracks paralleled to the coating/interlayer/substrate interfaces, and consequently prevented the delamination of diamond coating from the substrate.

\section{ATOMISTIC MODELING OF THE ANISOTROPIC STRENGTH OF TIC}

Atomistic modeling at density functional theory (DFT) level was conducted to study the underlying fracture mechanism of the crack orientations and confinement within the $\mathrm{TiC}$ interlayer. The calculations were performed using the Vienna $a b$ initio simulation package ${ }^{16,17}$ with a plane-wave cutoff energy of $440.0 \mathrm{eV}$. The ion-electron interactions were described by projector-augmented wave potentials. ${ }^{18}$ The exchange-correlation function was generalized gradient approximation of Perdew and Wang. ${ }^{19}$ The HellmannFeynman force of each atom was relaxed to below $0.05 \mathrm{eV}^{-1}$. Integrations in Brillouin zone were carried out on special $k$-points, which were generated by a $13 \times 13$ $\times 13$ grid for the cubic cell of bulk TiC and a $9 \times 9 \times 1$ grid for the slab models in surface energy calculations. 
TABLE I. Theoretical anisotropic strengths of TiC. $\gamma$ is the surface energy of the newly cleaved free surfaces. The TiC(111) surface energy is half the cleavage energy, which is sum of the Ti- and C-termed surface energies. $a_{0}$ is the equilibrium spacing between planes; $E$ is the Young's Modulus; $\sigma_{\max }$ is the theoretical strength. Values in parenthesis are from other calculations or experiments.

\begin{tabular}{|c|c|c|c|c|}
\hline Orientation & $\begin{array}{c}\gamma \\
\left(\mathrm{J} / \mathrm{m}^{2}\right)\end{array}$ & $\begin{array}{l}a_{0} \\
(\AA)\end{array}$ & $\begin{array}{c}E \\
(\mathrm{GPa})\end{array}$ & $\begin{array}{c}\sigma_{\max } \\
(\mathrm{GPa})\end{array}$ \\
\hline (111) & $\begin{array}{c}\text { Ti-termed: } 2.79\left(3.12^{\mathrm{a}}\right) \\
\text { C-termed: } 7.81\end{array}$ & 1.253 & 865.3 & 191.3 \\
\hline (100) & $\begin{array}{c}1.68 \\
\left(1.66,^{\mathrm{b}} 1.76,^{\mathrm{a}} 1.73^{\mathrm{c}}\right)\end{array}$ & 2.170 & $\begin{array}{l}495.8 \\
\left(497^{d}\right)\end{array}$ & 62.0 \\
\hline (110) & 3.53 & 1.534 & 478.9 & 105.0 \\
\hline
\end{tabular}

${ }^{\mathrm{a}}$ Reference 23.

${ }^{\mathrm{b}}$ Reference 22 .

${ }^{\mathrm{c}}$ Reference 24.

${ }^{\mathrm{d}}$ Reference 21.

The Griffith toughness of model I crack is related to the theoretical strength, which is the stress required to pull apart two planes in a perfect solid at absolute zero. ${ }^{20}$ The theoretical strength can be calculated by

$$
\sigma_{\max }=\sqrt{E \gamma / a_{0}}
$$

where $E$ is Young's modulus, $\gamma_{s}$ is the surface energy of the newly cleaved free surfaces, and $a_{0}$ is the equilibrium spacing between atomic planes computed based on the calculated lattice constant of $4.3395 \AA$. As mentioned above, $\mathrm{TiC}$ is strongly anisotropic, therefore $E$ was calculated separately for specific stresses normal to the low index surfaces of (111), (110), and (100), represented by $E_{(111)}, E_{(110)}$, and $E_{(100)}$, respectively. To obtain the anisotropic Young's modulus, a series of stretch strains of $0.5 \%, 1.0 \%, 1.5 \%$, and $2.0 \%$ were applied normal to these planes, then a linear leastsquare regression was performed to $H=H_{0}+\frac{1}{2} \varepsilon^{2} E$, where $H$ is the total energy with the applied strain of $\varepsilon ; H_{0}$ is the total energy of the unstrained state, and $E$ is the modulus. The calculated anisotropic elastic moduli were listed in Table I. Apparently, $E_{(111)}$ was about twice of $E_{(110)}$ and $E_{(100)}$, indicating the (111) orientation was much stronger than the other two orientations. Note that the calculated $E_{(100)}, 495.8 \mathrm{GPa}$, was very close to the experimental Young's modulus, 497 $\mathrm{GPa}$, along [100] direction of a single crystal $\mathrm{TiC}^{21}$ The surface energies of TiC were also very different along (111), (110), and (100) orientations. A series of surface slabs with different number of layers, $n$, separated by $10 \AA$ vacuum, were used to compute the surface energy, $\gamma_{s}$, through a linear least-square fitting to $H_{t}=(2 / n) \gamma_{s}+H_{b}$, where $H_{t}$ is the total energy per layer in the slab, and $H_{b}$ is the total energy per layer in the bulk. Since $\mathrm{TiC}$ has a $\mathrm{NaCl}$ structure, the (111) free surfaces can be either fully Ti-terminated or C-terminated, which shall have very different surface energies. The (110) and (100) surface have only one configuration, which is terminated stoichiometrically by $\mathrm{Ti}$ and $\mathrm{C}$. The calculated surface energies were listed in Table I and compared with experimental results and other DFT calculations. $^{22-24}$ As shown in Table I, among all the three TiC surfaces, the (100) surface has the lowest surface energy $\left(1.68 \mathrm{~J} \mathrm{~m}^{-2}\right)$.
The strong anisotropy of surface energy and elastic modulus of TiC implied the fracture toughness is also anisotropic. Here, we compared the theoretical strength along $\langle 111\rangle,\langle 110\rangle$, and $\langle 100\rangle$ directions calculated via Eq. (1) using the anisotropic Young's modulus and surface energies. Cleaving TiC will create two identical TiC(110) or (100) surfaces, thus the cleavage energy along (110) or (100) is the twice of the surface energy. Cleaving along TiC(111) will create one $\mathrm{Ti}$ and one $\mathrm{C}$ terminated surfaces, thus the cleavage energy is the sum of the surface energies of Ti- and C-termed surfaces, $10.60 \mathrm{~J} \mathrm{~m}^{-2}$, which is close to values reported before as $11.43 \mathrm{~J} \mathrm{~m}^{-2}$ (Ref. 22) and $11.26 \mathrm{~J} \mathrm{~m}^{-2}$. $^{24}$ The $\gamma_{(111)}$ used in Eq. (1) is therefore $5.30 \mathrm{~J} \mathrm{~m}^{-2}$. The computed ideal strengths along $\langle 111\rangle,\langle 110\rangle$, and $\langle 100\rangle$ were listed in Table I. Note that $\sigma_{\max (111)}$ is about three times, and $\sigma_{\max (110)}$ is about 1.7 times larger than $\sigma_{\max (100)}$, suggesting that it should be much easier for a crack to initiate and propagate along the (100) plane than along the (110) and (111) planes. The order of calculated theoretical strength is in good agreement with the indentation tests on single crystal TiC by Maerky et al., ${ }^{13}$ who found the fracture toughness for cracks along (100) plane was smaller than that along (110) plane, and the cracks along (111) plane were inactive. We also noticed that the calculated fracture energies are $3.36 \mathrm{~J} / \mathrm{m}^{2}$ along (100) and $7.06 \mathrm{~J} / \mathrm{m}^{2}$ along (110), which are very close to the critical strain energy release rates measured along (010)[100] and (110)[110], respectively, in Ref. 13. This quantitative agreement between our calculations and the experiments is better than the simple bond breaking model, in which the fracture energy is derived from the bond energies and number of bonds across the interface. ${ }^{13}$

Since the TiC interlayer forms between $\mathrm{Ti}$ and diamond, it is of great interest to compare the surface energies of $\mathrm{TiC}$ with pure $\mathrm{Ti}$ and diamond to identify the location of the cracks. Diamond (111) and Ti (0001) are orientations with the lowest surface energies among their low-index surfaces. DFT calculations showed that diamond (111)- $1 \times 1$ surface energy is $5.66 \mathrm{~J} \mathrm{~m}^{-2}$, 25 and $\mathrm{Ti}$ (0001) surface energy is $1.92 \mathrm{~J} \mathrm{~m}^{-2}$ (in the present study). Both are higher than the TiC(100) surface energy, $1.68 \mathrm{~J} \mathrm{~m}^{-2}$. This means that a cleavage in $\mathrm{TiC}$ along (100) plane requires less energy than that in $\mathrm{Ti}$ and diamond, and cracks are easier to initiate and propagate in $\mathrm{TiC}$ along the (100) plane than in $\mathrm{Ti}$ or diamond. The plasticity of Ti will further prevent crack initiation and propagation in the $\mathrm{Ti}$ substrate. This can explain why cracks were observed only in the $\mathrm{TiC}$ interlayer rather than in the $\mathrm{Ti}$ substrate in the three point bending tests.

\section{MICROCRACKS TOUGHENING}

The diamond coating was deposited on the Ti foil with a (0001) texture, as demonstrated by the higher (0002) peak in the XRD pattern compared with the simulated pattern of $\mathrm{Ti}$ powder with random orientations in Fig. 4(a). The intensity ratio between (0002) peak and (10 $\overline{1} 1)$ peak is 0.4 for a random orientation, while the ratio is 0.8 for the Ti foil. Thus, it is reasonable that $\mathrm{TiC}$ grew epitaxially on the (0001)textured $\mathrm{Ti}$ substrate with $\mathrm{TiC}(111) / \mathrm{Ti}(0001)$ orientation, because both the Ti-termed $\mathrm{TiC}(111)$ surface and the $\mathrm{Ti}(0001)$ 

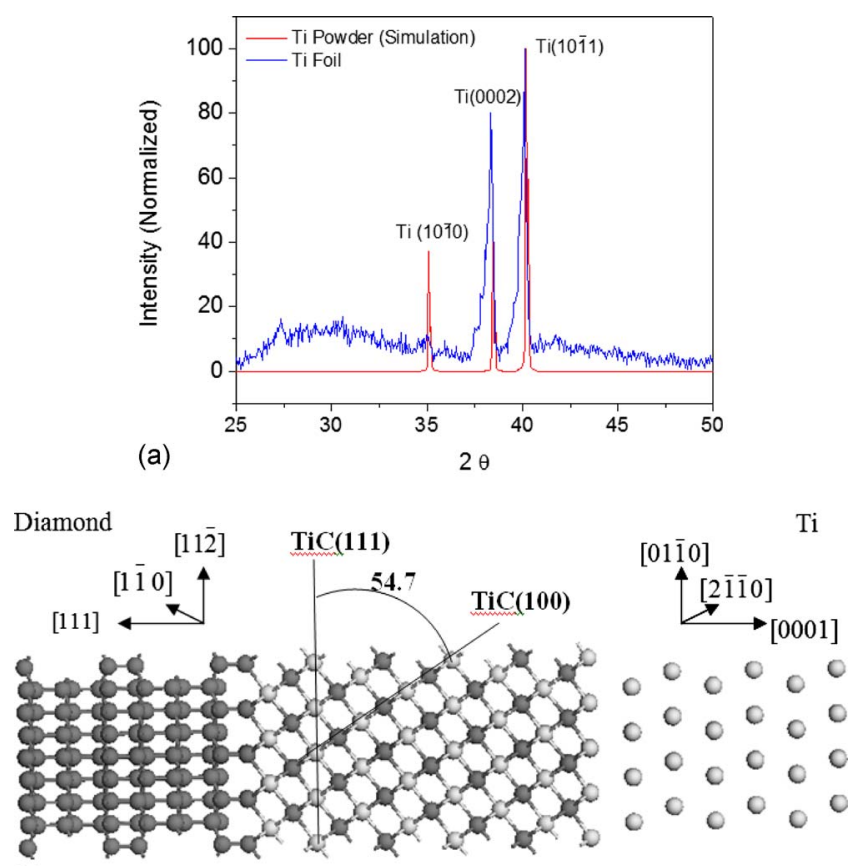

(b)

FIG. 4. (Color online) (a) XRD of Ti substrate before diamond deposition; (b) Schematic atomic structure of diamond (111)/TiC(111)/Ti(0001). Dark gray: carbon atoms; light gray: Ti atoms. The atomic planes are marked as projections onto the (110) plane of $\mathrm{TiC}$. The angle between $\mathrm{TiC}(100)$ and $\mathrm{TiC}(111)$ is $54.7^{\circ}$ as marked.

surface have similar hexagonal symmetries and the lattice mismatch between the two is only about $5 \%$. As the spontaneous formation of $\mathrm{TiC}$ during CVD involves diffusion of carbon atoms into the Ti substrate, we believe the TiC interlayer has (111) texture to conform to the $\mathrm{Ti}(0001)$ texture. It is expected that the diamond grown on $\mathrm{TiC}(111)$ would adopt the (111) orientation for similar reasons. A schematic atomic structure of the interface is shown in Fig. 4(b), where the $\mathrm{Ti}(0001), \mathrm{TiC}(111)$ and diamond(111) are all parallel to the coating surface. The angle between $\mathrm{TiC}\{111\}$ and $\{100\}$ is $54.7^{\circ}$, which is very close to the observed crack angles relative to the interface. Although the preferred (111) orientation in $\mathrm{TiC}$ is not obvious from the XRD [Fig. 2(c)], due to the thin thickness of $\mathrm{TiC}$ on a rather rough substrate, the aligned microcracks in Fig. 2(d) clearly indicated a preferred orientation of TiC phase.

The parallel $\sim 55^{\circ}$ orientation of the microcracks not only accommodates large strains during bending tests but also prevents microcracks bridging into macrocracks. Thus, they consequently avoid coating delamination. One might argue that cracks normal to the substrate would accommodate even more deformation. However, due to the cubic symmetry of the TiC crystal structure, such orientation would lead to identical weak $\{100\}$ planes also exist in parallel to the interface. Another feature of these cracks is that they close upon unloading, mainly due to the compressive residual stress created by the thermal expansion mismatch between TiC and Ti. When the microcracks reach the boundaries of the $\mathrm{TiC}$ interlayer, their further propagation becomes energetically unfavorable because they are confined between the strong diamond phase and the ductile Ti phase. Essentially, the parallel microcracks were constrained in TiC interlayer.

We note that the macroscopic XRD spectrum [shown in Fig. 2(c)] was unable to capture the (111) texture of the TiC interlayer, because the thickness of the $\mathrm{TiC}$ interlayer ( $\sim 0.2 \mu \mathrm{m}$ ) is well comparable with the surface roughness of the Ti substrate. Moreover, the peeling-off process before XRD may create (100) surface even though the TiC interlayer has (111) texture, because as demonstrated by the DFT calculations, the surface energy of (100) is much lower than (111). As a result, the data collected by XRD over macroscale in fact showed a more random orientation rather than a clear (111) texture. But at a smaller scale, such as the AFM scanning area, the relatively constant angles between the parallel cracks and the interface indicate the local orientation of the $\mathrm{TiC}$ interlayer is mainly determined by the texture of $\mathrm{Ti}$ substrate and should follow the mechanism proposed above. The control of TiC (111) orientation has been achieved on $\mathrm{Si}$ (001) substrate, as demonstrated by Mani et al. ${ }^{26}$

\section{DISCUSSIONS}

Based on experiments and DFT calculations, it was concluded that the structural and mechanical anisotropy of the TiC interlayer led to the oriented microcracks under the external load. The creation of these microcracks effectively absorbed strain energies and accommodates deformation. Their preferred orientation and confinement between the two interfaces prevented them bridging into macrocracks which could lead to film delamination. Furthermore, their self-closing effect under the compressive residual stress also benefited the coating reliability. This new "microcrack toughening" mechanism was manifested by the significant enhancement of the overall macroscopic interfacial toughness between diamond and $\mathrm{Ti}$ substrate, and the resulting improvement of coating adhesion. Microcracking is a toughening mechanism pioneered in brittle materials, ${ }^{27}$ such as ceramics and composites, where the microcracks are typically along the grain boundaries. ${ }^{28}$ Our experimental and modeling results revealed that the weak and strong crystallographic planes of a brittle phase (e.g., TiC) can be utilized to create preferably orientated microcracks. By confining these microcracks within the interlayer, the overall macroscopic toughness of the coating on the substrate is effectively enhanced and the integrated coating/interlayer/substrate system exhibits good adhesion. As shown in Fig. 1, even though the substrate as well as the coating went through severe deformation and many radically distributed microcracks in the coating were observed in and around the indent, no single piece of diamond coating delaminated in those areas from the substrate, which in fact indicated improved toughness since more strain energy had been dissipated by other mechanisms instead of interfacial fracture. The (111) orientated TiC phase can be formed by either on (0001) textured Ti during CVD diamond coating deposition, or in a separated deposition process such as biased sputtering on any substrate; thus, this toughing mechanism can be applied in more general cases. 


\section{CONCLUSIONS}

In summary, a new "microcrack toughening" mechanism has been proposed for enhancing the adhesion of an integrated coating/interlayer/substrate system, through confining multiple microcracks preferentially oriented away from the interface. The control of the microcrack orientation in the interlayer provides a new avenue to optimize the adhesion of thin-film coated structures and devices.

\section{ACKNOWLEDGMENTS}

The authors appreciate the insightful discussions with Dr. Y.-T. Cheng and Dr. Anil K. Sachdev. The calculations are performed at GM High Performance Computer Center and USC High Performance Computing Group.

${ }^{1}$ X. Xiao, B. W. Sheldon, Y. Qi, and A. K. Kothari, Appl. Phys. Lett. 92, 131908 (2008).

${ }^{2}$ Y. Qi, B. W. Sheldon, H. Guo, X. Xiao, and A. K. Kothari, Phys. Rev. Lett. 102, 056101 (2009).

${ }^{3}$ A. G. Evans, J. W. Hutchinson, and Y. Wei, Acta Mater. 47, 4093 (1999). ${ }^{4}$ M. Lane, Annu. Rev. Mater. Res. 33, 29 (2003).

${ }^{5}$ M. A. Taher, W. F. Schmidt, A. P. Malshe, E. J. Oles, and A. Inspektor, in Adhesion Aspects of Thin Films, edited by K. L. Mittal (VSP, Utrecht, 2001), Vol. 1.

${ }^{6}$ M. D. Drory and J. W. Hutchinson, Science 263, 1753 (1994).
${ }^{7}$ H. M. Liu and D. S. Haber, Diamond Chemical Vapor Deposition: Nucleation and Early Growth Stages (Noyes Publication, Park Ridge, NJ, 1995).

${ }^{8}$ D. R. Lide, Handbook of Chemistry and Physics, 71th ed. (CRC, Boca Raton, 1991).

${ }^{9}$ K. Schwarz, Crit. Rev. Solid State Mater. Sci. 13, 211 (1987).

${ }^{10}$ D. L. Price and B. R. Cooper, Phys. Rev. B 39, 4945 (1989).

${ }^{11}$ P. Blaha, J. Redinger, and K. Schwarz, Phys. Rev. B 31, 2316 (1985).

${ }^{12}$ S.-H. Jhi, J. Ihm, S. G. Louie, and M. L. Cohen, Nature (London) 399, 132 (1999).

${ }^{13}$ C. Maerky, M.-O. Guillou, J. L. Henshall, and R. M. Hooper, Mater. Sci. Eng., A 209, 329 (1996).

${ }^{14}$ X. D. Li, D. F. Diao, and B. Bhushan, Acta Mater. 45, 4453 (1997).

${ }^{15}$ Z. H. Xu and D. Rowcliffe, Surf. Coat. Technol. 161, 44 (2002).

${ }^{16}$ G. Kresse and J. Hafner, Phys. Rev. B 49, 14251 (1994).

${ }^{17}$ G. Kresse and J. Furthmüller, Comput. Mater. Sci. 6, 15 (1996).

${ }^{18}$ G. Kresse and J. Joubert, Phys. Rev. B 59, 1758 (1999).

${ }^{19}$ J. P. Perdew, J. A. Chevary, S. H. Vosko, K. A. Jackson, M. R. Pederson, D. J. Singh, and C. Fiolhais, Phys. Rev. B 46, 6671 (1992).

${ }^{20}$ I. LeMay, Principles of Mechanical Metallurgy (Elsevier, New York, 1982).

${ }^{21}$ K. E. Petersen, Proc. IEEE 70, 420 (1982).

${ }^{22}$ C. Ruberto and B. I. Lundqvist, Phys. Rev. B 75, 235438 (2007).

${ }^{23}$ A. Arya and E. A. Carter, J. Chem. Phys. 118, 8982 (2003).

${ }^{24}$ S. V. Dudiy and B. I. Lundqvist, Phys. Rev. B 69, 125421 (2004).

${ }^{25}$ Y. Qi and L. G. Hector, Jr., Phys. Rev. B 69, 235401 (2004).

${ }^{26}$ A. Mani, P. Aubert, F. Mercier, H. Khodja, C. Berthier, and P. Houdy, Surf. Coat. Technol. 194, 190 (2005).

${ }^{27}$ A. G. Evans, J. Am. Ceram. Soc. 73, 187 (1990).

${ }^{28}$ L. S. Sigl, Acta Mater. 44, 3599 (1996). 